\title{
MANEJO DE EPISTAXIS EN PACIENTES CON TERAPIA ANTITROMBÓTICA
}

\section{Management of epistaxis in patients with anti-thrombotic therapy}

\author{
María MARTÍN-BAILÓN ${ }^{1}$; Paula LÓPEZ-MESA², Cristina DIOS-LOUREIRO² \\ ${ }^{1}$ SACYL. Complejo Asistencial de Zamora. Servicio de Otorrinolaringología. Zamora. España. \\ ${ }^{2}$ SERGAS. Complejo Hospitalario Universitario de Santiago. Servicio de Otorrinolaringología. Santiago de \\ Compostela. España. \\ Correspondencia: mmartinbai@saludcastillayleon.es
}

Fecha de recepción: 22 de septiembre de 2020

Fecha de aceptación: 30 de octubre de 2020

Fecha de publicación: 6 de noviembre de 2020

Fecha de publicación del fascículo: 1 de junio de 2021

Conflicto de intereses: Los autores declaran no tener conflictos de intereses

Imágenes: Los autores declaran haber obtenido las imágenes con el permiso de los pacientes

Política de derechos y autoarchivo: se permite el autoarchivo de la versión post-print (SHERPA/RoMEO)

Licencia CC BY-NC-ND. Licencia Creative Commons Atribución-NoComercial-SinDerivar 4.0 Internacional

Universidad de Salamanca. Su comercialización está sujeta al permiso del editor

RESUMEN: Introducción y objetivo: la epistaxis es una de las urgencias otorrinolaringológicas más frecuentes. Una de sus causas son las alteraciones de la hemostasia provocadas por fármacos antitrombóticos. El objetivo del estudio es determinar si existen diferencias en el manejo de epistaxis entre pacientes con terapia antitrombótica y pacientes controles.

Método: estudio observacional analítico retrospectivo de pacientes ingresados en el servicio de Otorrinolaringología de un hospital de tercer nivel, entre enero de 2010 y diciembre de 2016. Se han recogido características epidemiológicas, clínicas y terapéuticas de los pacientes a través de su historia clínica electrónica y se ha realizado análisis estadístico comparativo entre los pacientes con terapia antitrombótica y pacientes controles.

Resultados: 85 pacientes analizados (74,1\% varones) con una edad media al diagnóstico de 66,7 años. El 49,4\% realizaban terapia antitrombótica. En todos los pacientes se realizó taponamiento anterior y en 16 pacientes, posterior. 37 pacientes precisaron cirugía endoscópica nasal. La embolización se realizó en cuatro pacientes. En el estudio estadístico comparativo no se han encontrado diferencias estadísticamente significativas entre ambos grupos en ninguna de las variables analizadas. 


\section{MANEJO DE EPISTAXIS EN PACIENTES CON TERAPIA ANTITROMBÓTICA MARTÍN-BAILÓN M ET AL.}

Discusión y conclusiones: las indicaciones actuales de terapia antitrombótica son muy amplias y por ello es frecuente atender epistaxis en este tipo de pacientes. Aunque no está claramente demostrado que la terapia antitrombótica por sí sola se asocie con sangrados más graves, creemos que es fundamental realizar un manejo multidisciplinar de estos pacientes con el fin de conseguir un adecuado control del sangrado sin necesidad de recurrir a intervenciones más agresivas.

PALABRAS CLAVE: Epistaxis; terapia antitrombótica; anticoagulante; taponamiento nasal

SUMMARY: Introduction and objective: epistaxis is one of the most frequent otorhinolaryngological emergencies. One of its causes is alterations in hemostasis caused by anti-thrombotic therapy. The aim of the study is to determine if there are differences in the management of epistaxis between patients with anti-thrombotic therapy and control patients.

Method: retrospective analytical observational study of patients admitted to the Otorhinolaryngology service of a tertiary hospital, between January 2010 and December 2016. Epidemiological, clinical and therapeutic characteristics of the patients have been collected through their electronic medical records and comparative statistical analysis was performed between patients with anti-thrombotic therapy and control patients.

Results: a total of 85 patients (74.1\% male) were analyzed. Mean age at diagnosis of 66.7 years. $49.4 \%$ were undergoing anti-thrombotic therapy. Anterior nasal packing was performed in all patients and posterior nasal packing in 16 patients. 37 patients required endoscopic nasal surgery. Embolization was performed in four patients. In the comparative statistical study, no statistically significant differences were found between both groups in any of the variables analyzed.

Discussion and conclusions: the current indications for anti-thrombotic therapy are very broad and therefore it is common to treat epistaxis in this type of patient. Although it has not been clearly demonstrated that anti-thrombotic therapy alone is associated with more serious bleeding, we believe that multidisciplinary management of these patients is essential in order to achieve adequate control of bleeding without the need for more aggressive interventions.

KEYWORDS: Epistaxis; anti-trhombotic therapy; anticoagulant; nasal packing

\section{INTRODUCCIÓN}

La epistaxis se define como una hemorragia con origen en las fosas o senos paranasales que se exterioriza a través de los orificios nasales o la boca [1]. Constituye una de las emergencias otorrinolaringológicas más comunes: se estima que el $60 \%$ de la población general sufrirá al menos un episodio a lo largo de su vida y el $6 \%$ necesitará asistencia médica en algún momento [1,2]; sin embargo, menos del $0.2 \%$ requerirá hospitalización [3]. Aunque su etiología se presume multifactorial, podemos realizar una clasificación etiológica en dos grupos: causas locales o causas sistémicas. Dentro de estas últimas, se recoge como causa importante de epistaxis las alteraciones en la hemostasia relacionadas con el uso de fármacos antitrombóticos (antiagregantes y/o anticoagulantes) [4]. En estudios previos se ha descrito que el $60 \%$ de los pacientes atendidos en un servicio de Urgencias por epistaxis recibían tratamiento antitrombótico [5]. El manejo de este tipo de pacientes supone siempre un reto para el otorrinolaringólogo: algunos estudios han demostrado estancias hospitalarias más prolongadas y necesidad de medidas hemostáticas más agresivas en los pacientes con tratamiento antiplaquetario y/o anticoagulante [6,7], así como mayor riesgo de recurrencia [8].

El objetivo de este estudio es determinar si los pacientes con terapia antitrombótica requieren medidas terapéuticas distintas y estancias 
hospitalarias más prolongadas que los pacientes sin dicho tratamiento.

\section{MATERIAL Y MÉTODO}

Se ha realizado un estudio observacional analítico retrospectivo de pacientes ingresados en el servicio de Otorrinolaringología del Complejo Hospitalario Universitario de Santiago de Compostela (población asignada de 447979 habitantes [9]) por epistaxis, entre enero de 2010 y diciembre de 2016, ambos inclusive. Para ello se revisó la historia clínica electrónica de estos pacientes, recogiendo sus características epidemiológicas, clínicas y terapéuticas. En concreto, las variables analizadas han sido sexo y edad al diagnóstico, comorbilidades, tiempo de hospitalización, tipo de terapia antibrombótica, tipo de tratamiento realizado incluyendo cauterización con nitrato de plata, taponamiento anterior, taponamiento posterior (tanto clásico con gasa como con sonda Foley o sonda de doble balón Bivona ${ }^{\circledR}$ ), y cirugía endoscópica nasal para realización de cauterización y/o ligadura de arteria esfenopalatina y embolización; necesidad de transfusión y número de concentrados de hematíes transfundidos, INR alterado y necesidad de reversión farmacológica, modificaciones realizadas en el tratamiento domiciliario y complicaciones.

El estudio estadístico ha consistido en un análisis comparativo entre los pacientes que recibían algún tipo de tratamiento antitrombótico (anticoagulante $\mathrm{o}$ antiagregante) y pacientes controles sin tratamiento. Se han comparado las variables tiempo de hospitalización, número de taponamientos anteriores realizados, necesidad de taponamiento posterior, necesidad de cirugía endoscópica nasal, necesidad de embolización y necesidad de transfusión y número de concentrados transfundidos. Se ha utilizado el soporte informático del programa SPSS 22.0 para Windows, aplicando el test $\mathrm{T}$ de Student para comparar variables cuantitativas entre dos poblaciones que siguen distribución normal y el test chi-cuadrado de Pearson para comparar variables cualitativas entre dos poblaciones de distribución normal; se han considerado estadísticamente significativo valores de p inferiores a 0,05.

\section{RESULTADOS}

En total se han incluido 85 pacientes, 63 varones $(74,1 \%)$ y 22 mujeres $(25,9 \%)$ con una edad media al diagnóstico de 66,7 $\pm 13,1$ años. El tiempo medio de hospitalización fue de 5,9 días (DS \pm 6.2 ). Las comorbilidades de estos pacientes se recogen en la Tabla 1; la patología asociada con mayor frecuencia fue la hipertensión arterial en el 48,2\% de los pacientes seguida de la fibrilación auricular en el $27,1 \%$. La hipertensión arterial fue más frecuente en el grupo de los pacientes con tratamiento antitrombótico (Tabla 2), siendo esta diferencia estadísticamente significativa ( 00,02$)$. Destacar que hasta 28 pacientes $(32,9 \%)$ presentaban más de una de estas comorbilidades. En cuanto al tratamiento, 42 pacientes $(49,4 \%)$ realizaban terapia con algún tipo de anticoagulante, antiagregante o asociación de varios (Tabla 3).

En lo referente a las medidas terapéuticas realizadas para controlar el sangrado nasal, en 22 pacientes se realizó cauterización con nitrato de plata; en todos los pacientes se realizó en al menos

Tabla 1. Comorbilidades de los pacientes estudiados

\begin{tabular}{|l|c|c|}
\hline \multicolumn{1}{|c|}{ Patología } & Total & Porcentaje \\
\hline Hipertensión arterial & 41 & $48,24 \%$ \\
Fibrilación auricular & 23 & $27,06 \%$ \\
Diabetes mellitus & 10 & $11,76 \%$ \\
Prótesis valvular mecánica & 10 & $11,76 \%$ \\
Accidente cerebrovascular & 5 & $5,88 \%$ \\
Marcapasos/DAI & 5 & $5,88 \%$ \\
Insuficiencia renal & 4 & $4,71 \%$ \\
Cardiopatía isquémica & 4 & $4,71 \%$ \\
Patología vascular periférica & 3 & $3,53 \%$ \\
Insuficiencia cardíaca & 3 & $3,53 \%$ \\
Traumatismo nasal & 2 & $2,35 \%$ \\
Patología hepática & 1 & $1,18 \%$ \\
\hline
\end{tabular}

*DAI: desfibrilador automático implantable 
Tabla 2. Frecuencia de comorbilidades en pacientes con tratamiento antitrombótico y controles

\begin{tabular}{|l|l|l|c|}
\hline & \multicolumn{1}{|c|}{$\begin{array}{c}\text { Pacientes a } \\
\text { tratamiento } \\
\text { antitrombótico } \\
(\mathrm{n}=42)\end{array}$} & $\begin{array}{c}\text { Controles } \\
(\mathrm{n}=43)\end{array}$ & $\mathrm{p}^{*}$ \\
\hline $\begin{array}{l}\text { Hipertensión } \\
\text { arterial }\end{array}$ & $\begin{array}{l}28 \text { pacientes } \\
(66,7 \%)\end{array}$ & $\begin{array}{l}13 \text { pacientes } \\
(30,2 \%)\end{array}$ & 0,02 \\
\hline $\begin{array}{l}\text { Diabetes } \\
\text { mellitus }\end{array}$ & 8 pacientes $(19 \%)$ & $\begin{array}{l}2 \text { pacientes } \\
(4,7 \%)\end{array}$ & 0,085 \\
\hline $\begin{array}{l}\text { Accidente } \\
\text { cerebrovascular }\end{array}$ & $\begin{array}{l}5 \text { pacientes } \\
(11,9 \%)\end{array}$ & 0 pacientes & 0,061 \\
\hline $\begin{array}{l}\text { Insuficiencia } \\
\text { renal }\end{array}$ & 3 pacientes $(7,1 \%)$ & $\begin{array}{l}1 \text { pacientes } \\
(2,3 \%)\end{array}$ & 0,592 \\
\hline $\begin{array}{l}\text { Cardiopatía } \\
\text { isquémica }\end{array}$ & 4 pacientes $(9,5 \%)$ & 0 pacientes & 0,119 \\
\hline $\begin{array}{l}\text { Insuficiencia } \\
\text { cardíaca }\end{array}$ & 3 pacientes $(7,1 \%)$ & 0 pacientes & 0,232 \\
\hline
\end{tabular}

* Se ha realizado prueba de chi-cuadrado para comparar proporciones en dos muestras independientes.

Tabla 3. Tratamiento antiagregante y/o anticoagulante

\begin{tabular}{|l|c|c|}
\hline \multicolumn{1}{|c|}{ Tratamiento } & Total & Porcentaje \\
\hline Monoterapia & 38 & $44,71 \%$ \\
\hline Acenocumarol & 23 & $27,06 \%$ \\
AAS & 9 & $10,59 \%$ \\
Clopidogrel & 2 & $2,35 \%$ \\
Warfarina & 2 & $2,35 \%$ \\
Enoxaparina & 1 & $1,18 \%$ \\
Dabigatrán & 1 & $1,18 \%$ \\
\hline Politerapia & 4 & $4,71 \%$ \\
\hline AAS + clopidogrel & 2 & $2,35 \%$ \\
AAS + acenocumarol & 1 & $1,18 \%$ \\
Cilostazol + triflusal & 1 & $1,18 \%$ \\
\hline Sin tratamiento & 43 & $50,59 \%$ \\
\hline
\end{tabular}

* AAS: ácido acetil-salicílico

una ocasión taponamiento anterior (en total se realizaron 192 taponamientos en los 85 pacientes), y 16 pacientes precisaron de la realización de taponamiento posterior. 37 pacientes fueron sometidos a cirugía endoscópica nasal y en ocho de ellos fue necesaria la reintervención. La embolización se realizó en cuatro pacientes. 21 de los pacientes $(24,7 \%)$ precisaron transfusión de concentrados de hematíes, con una media de 2,3 concentrados por paciente (rango 1-6). En seis pacientes fue necesario realizar reversión farmacológica de su tratamiento anticoagulante: cinco de ellos con vitamina $\mathrm{K}$ y uno con vitamina K y octaplex.

Las modificaciones realizadas en el tratamiento domiciliario fueron consensuadas con los servicios de Hematología y/o Cardiología en función de la patología de base del paciente: en los pacientes a tratamiento con acenocumarol, éste se sustituyó por enoxaparina en 16 pacientes, en un paciente por heparina sódica y en otro por AAS; en los cinco pacientes restantes no se realizaron modificaciones. En cuatro de los pacientes que tomaban AAS se suspendió temporalmente el tratamiento y en uno se sustituyó por enoxaparina. En el paciente anticoagulado con dabigatrán, éste se sustituyó por acenocumarol. En el paciente doblemente antiagregado con AAS y clopidogrel, se decidió suspender temporalmente este último. En el paciente antiagregado con clopidogrel y el que recibía tratamiento con cilostazol y disgren, se modificó temporalmente el tratamiento a enoxaparina. Por tanto, en el 62\% de los pacientes con tratamiento antitrombótico hubo que realizar cambios en este tratamiento para poder controlar el sangrado. 14 pacientes fueron valorados por el servicio de Medicina Interna por mal control de su hipertensión arterial ya conocida, ajustándose el tratamiento. En cinco pacientes se detectó tensión arterial elevada de forma mantenida durante el ingreso, siendo necesario el inicio de medicación antihipertensiva.

Como complicaciones durante el ingreso destacan un paciente que falleció por reagudización de su insuficiencia cardíaca de base, una parada cardiorrespiratoria recuperada en el contexto de deprivación alcohólica, un paciente con antecedente de cirrosis hepática y varices esofágicas que presentó sangrado digestivo alto con necesidad de realizar 


\section{MANEJO DE EPISTAXIS EN PACIENTES CON TERAPIA ANTITROMBÓTICA MARTÍN-BAILÓN M ET AL.}

traqueotomía de urgencia por imposibilidad para la intubación orotraqueal, y un paciente que se complicó con un tromboembolismo pulmonar. Mencionar el caso de un paciente que falleció por hemorragia cerebral espontánea dos meses después de haber sido dado de alta de nuestro servicio; este paciente estaba anticoagulado con warfarina por fibrilación auricular, era hipertenso y diabético, y el sangrado nasal que motivó el ingreso en nuestro servicio fue controlado con taponamiento anterior y no se realizó ninguna modificación en su tratamiento domiciliario.

En la Tabla 4 se exponen las características de los pacientes que recibían algún tipo de tratamiento anticoagulante $y / o$ antiagregante frente a las de los pacientes que no recibían ningún tipo de tratamiento. Al realizar el estudio estadístico comparativo entre ambos grupos no se han encontrado diferencias estadísticamente significativas entre ambos grupos en cuanto a tiempo de hospitalización (5,67 días vs 6,07 en el grupo control), número de taponamientos anteriores realizados (2,3 taponamientos por paciente vs 2,2$)$, necesidad de taponamiento posterior (8 pacientes en ambos grupos), necesidad de CENS (15 pacientes vs 23 ), necesidad de embolización ( 3 pacientes vs 1), necesidad de transfusión (9 pacientes vs 12) ni número de concentrados de hematíes transfundidos $(2,2$ vs 2,3$)$.

\section{DISCUSIÓN}

La epistaxis supone un motivo frecuente de asistencia a los servicios de urgencias. En el estudio epidemiológico sobre epistaxis realizado en el Complejo Hospitalario Universitario de Santiago de Compostela en 1999 por Vaamonde y colaboradores [10] se obtuvo una incidencia de 6 casos por 100000 habitantes/años; la epistaxis suponía 0,6 de cada 200 consultas en el servicio de urgencias y el 13,3\% de las urgencias otorrinolaringológicas. Por otro lado, los tratamientos que alteran la hemostasia (anticoagulantes y antiagregantes) se siguen considerando un factor predisponente para la aparición de epistaxis [3]. Aunque no es posible determinar la prevalencia real de pacientes anticoagulados en España, algunos estudios la han estimado en 1,3 casos por cada 100 habitantes [11]. Esta prevalencia es probable que siga aumentando en los próximos años debido al envejecimiento poblacional y al incremento de las indicaciones para terapia anticoagulante. Lo mismo ocurre con el tratamiento antiagregante ya que también ha aumentado la supervivencia de las patologías que

Tabla 4. Diferencias entre pacientes con tratamiento antitrombótico y controles

\begin{tabular}{|l|l|l|c|}
\hline & \multicolumn{1}{|c|}{ Pacientes a tratamiento antitrombótico $(\mathrm{n}=42)$} & \multicolumn{1}{|c|}{ Controles $(\mathrm{n}=43)$} & $\mathrm{p}^{*}$ \\
\hline Edad & $71,9 \pm 9,7$ & $61,6 \pm 14,1$ & 0,128 \\
\hline Sexo & $78,6 \%$ varones $(33 \mathrm{H} / 9 \mathrm{M})$ & $70 \%$ varones $(30 \mathrm{H} / 13 \mathrm{M})$ & 0,354 \\
\hline Hospitalización (días) & $5,7 \pm 3,4$ & $6,1 \pm 8,1$ & 0,454 \\
\hline$N^{\circ}$ taponamientos anteriores por paciente & 2,3 & 2,2 & 0,480 \\
\hline Necesidad de taponamiento posterior & 8 pacientes $(19 \%)$ & 8 pacientes $(18,6 \%)$ & 1,000 \\
\hline CENS** & 15 pacientes $(35,7 \%)$ & 23 pacientes $(53,5 \%)$ & 0,153 \\
\hline Embolización & $3(7,1 \%)$ & $1(2,3 \%)$ & 0,360 \\
\hline Transfusión & 9 pacientes $(21,4 \%)$ & 12 pacientes $(27,9 \%)$ & 0,659 \\
\hline No $^{*}$ concentrados de hematíes & 2,2 concentrados/paciente & 2,3 concentrados/paciente & 0,214 \\
\hline
\end{tabular}

Se ha calculado desviación estándar media o porcentaje, según corresponde en cada caso.

${ }^{*}$ Se ha realizado T-student, prueba de chi-cuadrado de Pearson o test exacto de Fisher según corresponde en cada caso. ${ }^{\star *}$ CENS: cirugía endoscópica nasal. 
requieren dicha medicación. En cuanto a la morbilidad asociada a estos tratamientos, la hemorragia es la más frecuente, sobre todo a nivel cutáneomucoso. En el estudio multicéntrico realizado por Navarro [12] en pacientes anticoagulados con acenocumarol, un $57,75 \%$ de ellos presentaron epistaxis como complicación hemorrágica, la mayoría leve o moderada, pero un pequeño porcentaje $(1,14 \%)$ fueron graves.

El objetivo principal de este estudio es determinar si el manejo de los pacientes ingresados por epistaxis con tratamiento antitrombótico difiere de los pacientes que no reciben esta terapia. No se han encontrado diferencias estadísticamente significativas entre ambos grupos en ninguno de los parámetros analizados. Sin embargo, estos resultados deben tomarse con cautela ya que se trata de un estudio retrospectivo y en el análisis comparativo no se ha tenido en cuenta la presencia de otras comorbilidades (hipertensión arterial, diabetes, insuficiencia renal, etc.) que no solo pueden influir en la gravedad de la epistaxis si no también prolongar por sí solas la estancia hospitalaria.

Nuestros resultados están en consonancia con otros estudios publicados sobre el tema. Ya en el estudio publicado en 1997 por Srinivasan [13] no se encontraron diferencias estadísticamente significativas en la estancia hospitalaria de los pacientes anticoagulados con warfarina en comparación con pacientes controles. En un estudio reciente, Buchberger y colaboradores [14] concluyen que los anticoagulantes orales no se asocian con episodios de epistaxis más complicados y graves, aunque si pueden ser más recurrentes. Sin embargo, en el estudio realizado por Smith [15] si encontraron que los pacientes anticoagulados con warfarina eran más propensos a requerir medidas más agresivas (como el taponamiento nasal posterior o la cirugía), y precisaban de estancias hospitalarias más prolongadas en comparación con los pacientes no anticoagulados. Del mismo modo, García y colaboradores compararon pacientes anticoagulados con dabigatrán frente a pacientes anticoagulados con acenocumarol y pacientes controles; en este estudio si se obtuvieron diferencias entre los tres grupos, con mayor tasa de ingreso hospitalario y necesidad de medidas terapéuticas más agresivas en el grupo del dagigatrán [16]. En cuanto a las diferencias entre los distintos agentes anticoagulantes y antiagregantes, otro estudio reciente [17] llega a la conclusión de que los pacientes en tratamiento con los nuevos anticoagulantes (inhibidores del factor $\mathrm{Xa}$ ) tienen menos episodios de epistaxis antes del ingreso, menor necesidad de control endoscópico quirúrgico y menor tasa de reingreso que los otros grupos. El estudio de L'Huillier [18] también mostró menor estancia hospitalaria en el grupo de los nuevos anticoagulantes orales, así como sangrados más leves. Del mismo modo, Sauter ha reportado menor necesidad de ingreso y estancias hospitalarias más cortas en paciente a tratamiento con rivaroxabán en comparación con antagonistas de vitamina $\mathrm{K}$, probablemente debido a una mayor tendencia a las epistaxis anteriores en el primer grupo [19]. En contraposición, Send [20] no encontró en su estudio ninguna diferencia significativa entre anticoagulantes directos y terapia antivitamina K. Además, hay que tener en cuenta que el manejo de las hemorragias relacionadas con estos nuevos anticoagulantes puede ser difícil ya que actualmente no existen antídotos que neutralicen el efecto de muchos de ellos [21] o no se encuentran disponibles en todos los hospitales. En lo que sí coinciden todos los autores es en la necesidad de un manejo multidisciplinar en estos pacientes para llevar a cabo las modificaciones en el tratamiento anticoagulante más adecuadas en cada caso [13-20].

\section{CONCLUSIONES}

La epistaxis es una de las urgencias otorrinolaringológicas más comunes. A pesar de que en la mayoría de las ocasiones se trata de episodios leves, no debemos olvidar que en algunos casos suponen un auténtico riesgo vital para el paciente. 


\section{MANEJO DE EPISTAXIS EN PACIENTES CON TERAPIA ANTITROMBÓTICA \\ MARTÍN-BAILÓN M ET AL.}

Las indicaciones actuales de terapia antitrombótica son muy amplias y por ello es cada vez más frecuente atender epistaxis en pacientes con este tipo de tratamiento. Además suele tratarse de pacientes con múltiples patologías asociadas, cuyo manejo multidisciplinar es imprescindible. Aunque no está claramente demostrado que la terapia antitrombótica por sí sola se asocie con sangrados más graves, creemos que es fundamental realizar una revisión exhaustiva del tratamiento domiciliario de estos pacientes por los especialistas que corresponda (Hematología, Cardiología y/o Medicina Interna) y realizar las modificaciones precisas en cada caso. Estas medidas pueden ser suficientes en algunos casos para conseguir un adecuado control del sangrado sin necesidad de recurrir a intervenciones más agresivas (cirugía endoscópica nasal o embolización).

\section{BIBLIOGRAFÍA}

1. Ramírez-Sabio J, De Paula-Berneta C, Marco Algarra J. Epistaxis. En: Suárez C, Gil-Carcedo, LM, Marco J, Medina J, Ortega P, Trinidad J. Tratado de Otorrinolaringología y Cirugía de Cabeza y Cuello. $2^{\mathrm{a}}$ ed. Madrid: Editorial Médica Panamericana; 2007. p. 637-35.

2. Rudmik L, Smith T. Management of intractable spontaneous epistaxis. Am J Rhinol Allergy. 2012; 26(1):55-60.

3. Villwock JA, Jones K. Recent trends in epistaxis management in the United States. 20082010. JAMA Otolaryngol Head Neck Surg. 2013;139(12):1279-84.

4. Yau, S. An update on epistaxis. Aust Fam Physician. 2015;44(9):653-6.

5. Musgrave KM, Powell J. A systematic review of anti-thrombotic therapy in epistaxis. Rhinology. 2016;54(4):292-391.

6. Smith J, Siddiq S, Dyer C, Rainsbury J, Kim D. Epistaxis in patients taking oral anticoagulant and antiplatelet medication: prospective cohort study. J Laryngol Otol. 2011;125(01):38-42.

7. García-Cabo P, Fernández-Vañes L, Pedregal D, Menéndez Del Castro M, Murias E, Vega P,
Llorente JL, Rodrigo JP, López F. Manejo de las epistaxis graves yo refractarias. Acta Otorrinolaringol Esp. 2019;70(4):185-91.

8. Gomes P, Salvador P, Lombo C, Caselhos S, Fonseca R. Role of age and anticoagulants in recurrent idiopathic epistaxis. Acta Otorrinolaringol Esp. 2019 Sep 11. pii: S0001-6519(19)30119-0. [Epub ahead of print].

9. Servizo Galego de Saúde (s.f.). Área Sanitaria de Santiago de Compostela e Barbanza. Entorno. Recuperado el 26 de octubre de 2020 de https:// xxisantiago.sergas.gal/DXerais/1008/Poblacion .pdf

10. Vaamonde-Lago P, Martín-Martín C, CajadeFrías J, Mínguez-Beltrán I, Lechuga-García MR, Frade-González C, Bartual-Magro J, LabellaCaballero T. Datos epidemiológicos sobre la epistaxis: estudio hospitalario y revisión de la literatura. Acta Otorrinolaringol. Gallega 2003;4:1-8. ISSN-e 2340-3438.

11. Boned-Ombuena A, Pérez-Panadés J, LópezMaside A, Miralles-Espí M, Guardiola Vilarroig S, Adam Ruiz D, Zurriaga O. Prevalencia de la anticoagulación oral y calidad de su seguimiento en el ámbito de la atención primaria: estudio de la Red Centinela Sanitaria de la Comunitat Valenciana. Aten Primaria. 2017;49(9):534-48.

12. Navarro JL, Cesar JM, Fernández MA, Fontcuberta J, Reverter JC, Gol-Freixa J. Morbilidad y mortalidad en pacientes con tratamiento anticoagulante oral. Rev Esp Cardiol. 2007;60(12): 1226-32.

13. Srinivasan V, Patel H, John DG, Worsley A. Warfarin and epistaxis: should warfarin always be discontinued? Clin Otolaryngol Allied Sci. 1997;22(6):542-4.

14. Buchberger AMS, Baumann A, Johnson F, Peters N, Piontek G, Storck K, Pickhard A. The role of oral anticoagulants in epistaxis. Eur Arch Otorhinolaryngol. 2018;275(8):2035-43.

15. Smith J, Siddiq S, Dyer C, Rainsbury J, Kim D. Epistaxis in patients taking oral anticoagulant and antiplatelet medication: prospective cohort study. J Laryngol Otol. 2011;125(1):38-42.

16. García Callejo FJ, Bécares Martínez C, Calvo González J, Martínez Beneyto P, Marco Sanz 


\section{MANEJO DE EPISTAXIS EN PACIENTES CON TERAPIA ANTITROMBÓTICA MARTÍN-BAILÓN M ET AL.}

M, Marco Algarra J. Epistaxis y dabigatrán, nuevo anticoagulante oral no antagonista de la vitamina K. Acta Otorrinolaringológica Esp. 2014;65(6):346-54.

17. Glikson E, Chavkin U, Madgar O, Sagiv D, Nakache G, Yakirevitch A, Wolf M, Alon E. Epistaxis in the setting of antithrombotic therapy: a comparison between factor Xa inhibitors, warfarin, and antiplatelet agents. Laryngoscope. 2019; 129(1):119-23.

18. L'Huillier V, Badet C, Tavernier L. Epistaxis complicating treatment by anti-vitamin $\mathrm{K}$ and new oral anticoagulants. Eur Ann Otorhinolaryngol Head Neck Dis. 2018;135(4): 231-5.
19. Sauter TC, Hegazy K, Hautz WE, Krummrey G, Ricklin ME, Nagler M, Borner U, Exadaktylos AK. Epistaxis in anticoagulated patients: fewer hospital admissions and shorter hospital stays on rivaroxaban compared to phenprocoumon. Clin Otolaryngol. 2018;43(1):103-8.

20. Send T, Bertlich M, Horlbeck F, Schafigh D, Freytag S, Eichhorn KW, Gräff I, Bootz F, Jakob M. Management and outcome of epistaxis under direct oral anticoagulants: a comparison with warfarin. Int Forum Allergy Rhinol. 2019;9(1):120-124

21. Mateo J. Nuevos anticoagulantes orales y su papel en la práctica clínica. Rev Esp Cardiol Supl. 2013;13(3):33-41. 